\title{
A Neonate With TGA and Restrictive Foramen Ovale: How to Apply Ordinary Catheterization Through the Umbilical Vein to Rescue the Neonate, a Case Report
}

\author{
Renata Bokiniec $^{\mathrm{a}, \mathrm{c}}$, Katarzyna Kufel ${ }^{\mathrm{a}}$, Maria K. Borszewska-Kornacka ${ }^{\mathrm{a}}$, Joanna Szymkiewicz-Dangel ${ }^{\mathrm{b}}$
}

\begin{abstract}
Transposition of the great vessels (TGA), particularly with a restrictive foramen ovale (FO), constitutes a direct threat to neonatal life in the first hours of life. Poor mixing of oxygenated and deoxygenated blood leads to severe cyanosis and death. Administration of prostaglandin E1 (Alprostadil) to maintain the patency of the arterial duct allows an increase of the pulmonary blood flow and its return to the left atrium. In cases of TGA with restricted interatrial communication, higher pressure in the left atrium causes closing of the FO flap and better mixing cannot be achieved. In such cases, urgent balloon atrial septostomy (BAS - Rashkind procedure) is the method of choice. It enables mixing of the blood and survival of the child until the surgical procedure can be performed. We present the case of a neonate in whom the FO flap was opened by introducing an ordinary catheter (3.5 French) via the umbilical vein. The procedure was performed, with good effect, at a neonatal center that had no facilities for invasive cardiology treatment.
\end{abstract}

Keywords: TGA; Restrictive patent foramen ovale; Umbilical vein catheterization

\section{Introduction}

The immediate management of the neonate with transposition of the great arterial vessels (TGA), after birth, is based upon the achievement of safe level of oxygenation and stabilization of the circulatory and respiratory systems. A procedure which ensures acceptable oxygenation in the neonate with TGA and restrictive foramen ovale (FO) is that of balloon atrial septo-

Manuscript accepted for publication July 07, 2014

${ }^{a}$ Neonatal and Intensive Care Department, Medical University of Warsaw, Poland

berinatal Cardiology Unit, 2nd Department of Obstetrics and Gynecology, Medical University of Warsaw, Poland

${ }^{\mathrm{c} C o r r e s p o n d i n g ~ A u t h o r: ~ R e n a t a ~ B o k i n i e c, ~ N e o n a t a l ~ a n d ~ I n t e n s i v e ~ C a r e ~ D e-~}$ partment, Medical University of Warsaw, ul. Karowa 2, 00-315 Warszawa, Poland. Email:r.bokiniec@wp.pl

doi: http://dx.doi.org/10.14740/jmc1853w stomy (BAS), which is performed as soon as possible after birth. It provides for the widening of the FO, which significantly improves the mixing of blood at the atrial level. Because of the direct threat to survival, the Rahskind procedure is often performed at the bedside, under echocardiographic control using the special balloon-tipped catheter inserted through the umbilical vein or the femoral vessels into the right and then the left atrium [1]. In Poland this procedure is performed in the catheterization laboratory. This report concerns the case of a neonate with TGA and restrictive FO, in whom the insertion of an ordinary catheter into the FO through the umbilical vein resulted in an increase in oxygen saturation to an acceptable level, which allowed for the safe transport of the child to a cardiac center.

\section{Case Report}

A male neonate was born vaginally to a mother (gravida 3, para 2) at 39 weeks of gestation. The pregnancy was complicated by insulin-dependent gestational diabetes and hypertension. At 20 weeks of gestation, following obstetric ultrasonography, a congenital heart defect - transposition of the great arteries was suspected in the fetus. The diagnosis was confirmed at the fetal cardiology referral center at 21 weeks of gestation. The blood flow through the FO was already bidirectional, without severe restriction. Fetal monitoring occurred every 5 weeks. Restrictive FO with normal flow across the arterial duct was diagnosed at 37 weeks of gestation (Fig. 1). This finding was important for the neonatology team in the tertiary perinatal referral center because it did not have any facilities for invasive cardiology treatment. Clear amniotic fluid flowed $11 \mathrm{~h}$ before the delivery. Normal physiological flora was cultured from the genital tract during pregnancy.

The neonate was born in a poor condition (gender: male, birth weight: 2,860 g, the Apgar scores at 1 and 5 min of life were 2 and 5 , respectively). He required intubation at $2 \mathrm{~min}$ of life and ventilation with Neopuff was commenced with an oxygen concentration of $0.6-0.3$. Treatment with prostaglandin E2 in the dose of $0.02 \mu \mathrm{g} / \mathrm{kg} / \mathrm{min}$ was administered immediately after birth. At 3 and 5 min after birth saturation was $20 \%$ and $45 \%$, respectively. He was transferred to the neonatal intensive care unit (NICU) and ventilated mechanically. In the NICU he was ventilated in the SIMV mode with a frequency 


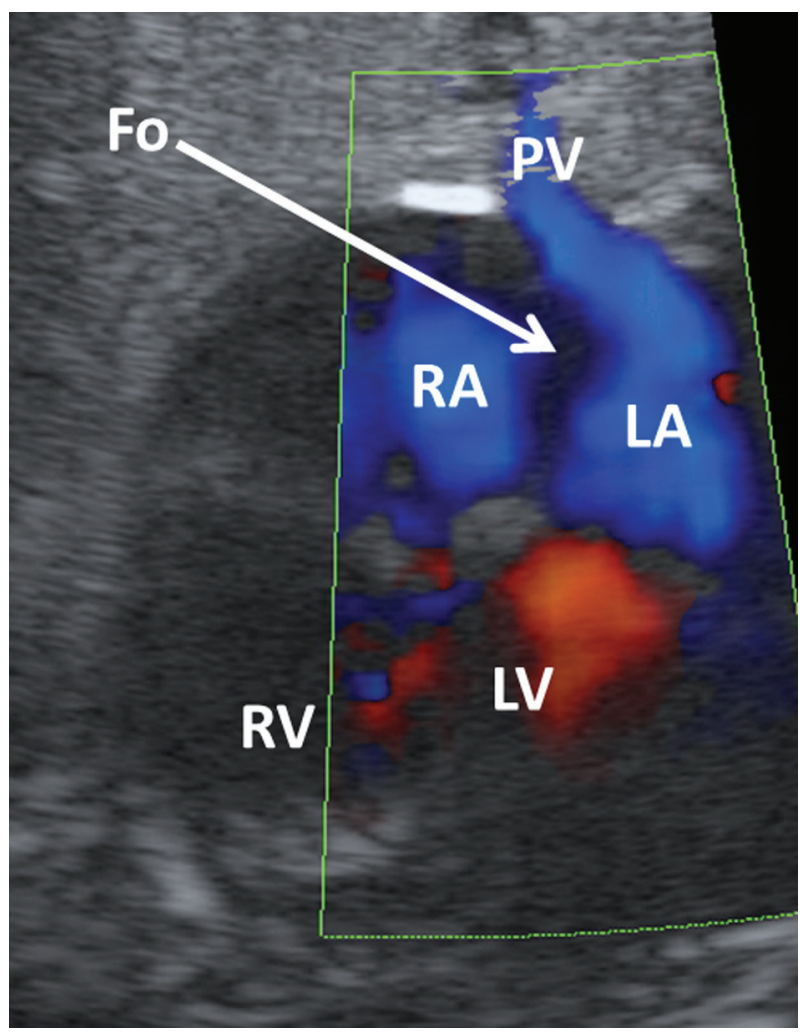

Figure 1. Foramen ovale in a fetus with TGA - flat interatrial septum and no mixing of the blood is seen.

of 45, PIP $+18, \mathrm{PEEP}+5, \mathrm{FiO}_{2}$ 1.0. A 3.5 French cannula was inserted into the umbilical vein and a 2.5 French cannula was inserted into the umbilical artery. During cannulation the saturation was low between $40 \%$ and $50 \%$. The neonatologist on call had a 10-year experience in neonatal echocardiography, and was able to confirm the diagnosis of the restricted FO. The following signs were detected during emergency echocardiography: 1) small left to right shunt across the FO; 2) blood flow velocity was $1.4 \mathrm{~m} / \mathrm{s}$, indicating an interatrial gradient $>7 \mathrm{~mm}$ $\mathrm{Hg}$; 3) enlarged left atrium; 4) interatrial septum was herniated to the right. The decision was made to slide the umbilical vein catheter deeper into the left atrium. We hoped that after this maneuver the FO flap will be pushed toward the left atrium and a bigger opening between the atria would enable better blood mixing at this level. When the catheter was at a depth of $15 \mathrm{~cm}$, the saturation increased to $70-80 \%$.

Echocardiographic monitoring during cannulation confirmed the position of the catheter in the left atrium. When an attempt was made to place the catheter in the correct position (the tip of the cannula should be in the inferior vena cava at 11 $\mathrm{cm}$ ) a sudden drop in saturation to $50 \% \mathrm{SpO}_{2}$ was observed. Therefore, since it was not possible to perform an emergency BAS, the cannula was left at $15 \mathrm{~cm}$ in the left atrium. Saturation was stabilized at $75 \%$, with continuous Alprostadil administration. The position of the catheter was also confirmed radiologically (Fig. 2). At the end of the first hour of life the saturation of the neonate was $75 \%$. In the second hour of life,

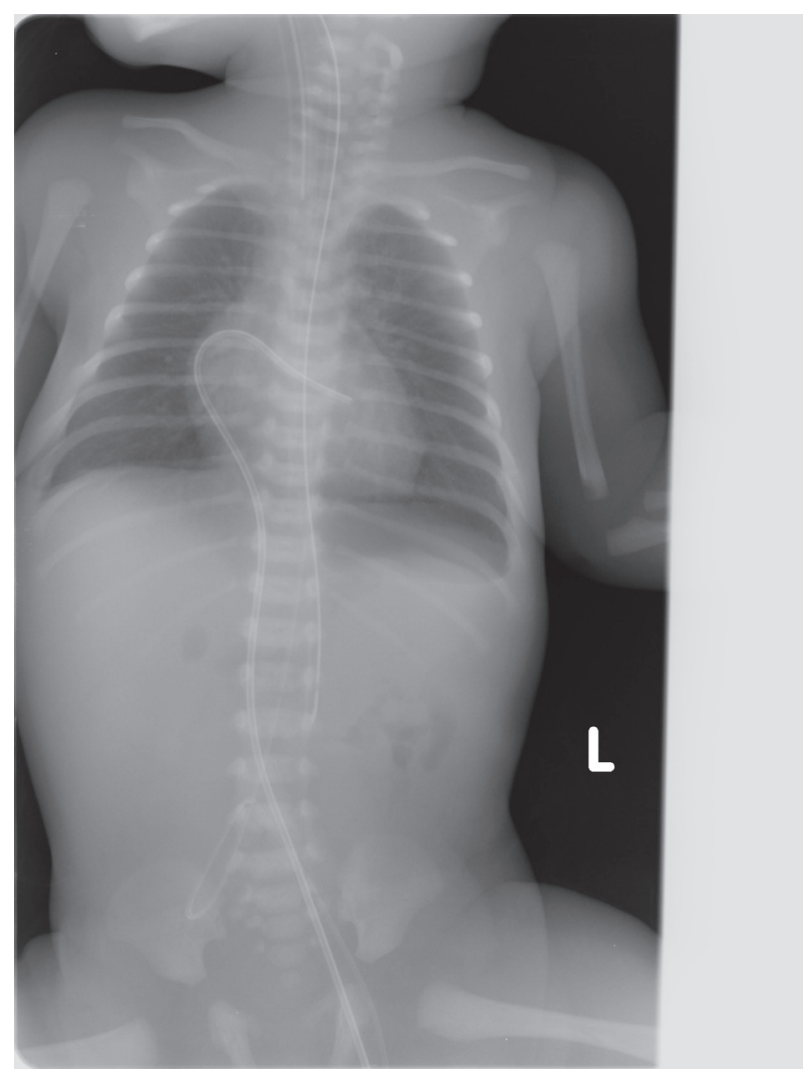

Figure 2. Radiological monitoring of the position of the catheter in the left atrium after traversing the foramen ovale.

the child was transferred by neonatal ambulance to a cardiology center $15 \mathrm{~km}$ away. BAS was performed the next day. The saturation was satisfactory; however, the neonate needed intensive care until the Rashkind procedure was performed. The arterial switch operation was performed on the 20th day of life, without complications.

\section{Discussion}

Transposition of the great arteries is the most frequent cyanotic cardiac defect in newborns. TGA, particularly with a restrictive FO, is a life-threatening condition for the neonate. Differences in the appearance of the FO flap in the fetus with TGA were already described in literature [2]. The FO becomes restricted at the end of pregnancy in fetuses with TGA, therefore they need close monitoring [2]. Restrictive FO was described in many cardiac lesions in the fetus [3], and it is clear that this diagnosis is much more difficult in the fetus with TGA when compared with those who have a hypoplastic left heart syndrome. Commonly used criteria such as high velocity left to right shunt across the FO or abnormal flow pattern in the pulmonary veins, are not helpful in the fetus with TGA.

In the present case, the atrial septum was flat - the septum primum was attached to the septum secundum and there was no flow across the FO at the end of pregnancy. This feature resulted in the very poor condition of the neonate. In the first 
neonatal echocardiogram immediately after delivery there was a narrow left to right shunt across the FO. The blood flow velocity was $1.4 \mathrm{~m} / \mathrm{s}$, which indicated an almost $8 \mathrm{~mm} \mathrm{Hg}$ pressure gradient between the atria. The interatrial septum was deflected toward the right atrium, the left atrium was enlarged, and both signs indicated a restrictive FO. Recently, Punn et al [2] described the significant features of restrictive FO in the fetus including the aforementioned appearance.

The aim of prenatal diagnosis of critical congenital heart defects is to improve the outcome and reduce mortality and morbidity $[3,4]$. TGA is not truly a duct-dependent congenital heart defect, as the main level for successful mixing is the FO. Administration of prostaglandin E1 (Alprostadil) in order to maintain the patency of the arterial tract allows an increase in the pulmonary flow and in its return to the left atrium, but in neonates with restrictive FO it does not improve blood oxygenation. Therefore, urgent BAS, which provides mixing of the blood, is the method of choice. Rashkind [1] was the first to describe this technique. The Rashkind catheter is introduced through the femoral and inferior cava vein to the right atrium and then it crosses the $\mathrm{FO}$ and reaches the left atrium. A balloon is inflated in the left atrium and pulled back to tear the FO flap. The procedure has been performed under radiological monitoring for many years. Echocardiographic monitoring during BAS has been used since the mid-eighties [5] Such monitoring enables this procedure to be performed in the NICU through the umbilical, instead of the femoral vein. In those centers with 24-h access to invasive cardiology services, BAS may be performed even within 45 min after birth [5]. Such facilities are not always available and neonates with critical heart defects may be born in specialized perinatology centers, but will require transfer to cardiac units. In babies with TGA and restrictive FO this delay can be life-threatening, so an alternative emergency procedure which permits transfer of the neonate in a good condition is necessary. Therefore, after observing the spectacular improvement in the oxygenation of our patient, we decided to leave the ordinary intravenous catheter, used in the catheterization of the umbilical vein, in the left atrium. This resulted in an increase of blood oxygenation within the first hour of life. The ordinary intravenous catheter did not tear the valve of the FO, but most probably pushed it apart, providing for an improvement of the flow of blood between the atria and hence oxygenation, which made the safe transport of the neonate possible.

\section{References}

1. Rashkind WJ, Miller WW. Creation of an atrial septal defect without thoracotomy. A palliative approach to complete transposition of the great arteries. JAMA. 1966;196(11):991-992.

2. Punn R, Silverman NH. Fetal predictors of urgent balloon atrial septostomy in neonates with complete transposition. J Am Soc Echocardiogr. 2011;24(4):425-430.

3. Donofrio MT, Bremer YA, Moskowitz WB. Diagnosis and management of restricted or closed foramen ovale in fetuses with congenital heart disease. Am J Cardiol. 2004;94(10):1348-1351.

4. Maeno YV, Kamenir SA, Sinclair B, van der Velde ME, Smallhorn JF, Hornberger LK. Prenatal features of ductus arteriosus constriction and restrictive foramen ovale in d-transposition of the great arteries. Circulation. 1999;99(9):1209-1214.

5. Baker EJ, Allan LD, Tynan MJ, Jones OD, Joseph MC, Deverall PB. Balloon atrial septostomy in the neonatal intensive care unit. Br Heart J. 1984;51(4):377-378. 